IJßER

ISSN: 2149-5939

\title{
The level of burnout experienced by teachers
}

\author{
Kıvanç Bozkuş ${ }^{1}$
}

Received Date: 05/ 10/2017

Accepted Date: 28/ 12 / 2017

\begin{abstract}
In this research, which was conducted to determine burnout experienced by teachers working at public schools in Kütahya, Turkey data collected from 386 teachers were analyzed. Results indicate that almost half of teachers experience burnout. Great number of teachers feel tired at least occasionally. Female teachers feel tired more than their male counterparts. Male teachers feel trapped, worthless and unsuccessful more than their female colleagues. Teachers working at technical high schools experience burnout more than other teachers. Reduction of teacher workload, exploration of why teachers experience burnout and generating preventive measures from research findings were suggested.
\end{abstract}

Keywords: Teacher, school, burnout

\section{Introduction}

People have negative feelings when their expectations do not comply with their work life (Schaufeli \& Enzmann, 1998). This psychological syndrome is called as burnout and it results in failure, dissatisfaction, and low energy so that it reduces work efficiency and productivity (Freudenberger, 1974). Burnout is experienced more in professions that have intensive human relations (Maslach \& Jackson, 1981; Maslach, 2003). Therefore, Maslach and Jackson associate burnout with these professions and define it as "a syndrome of emotional exhaustion, depersonalization, and reduced personal accomplishment that can occur among individuals who do 'people work' of some kind" (1986, p. 1). Teachers are exposed to burnout more than individuals working at other professions (De Heus \& Diekstra, 1999). Work environment, for this reason, acts as a main factor affecting burnout (Maslach, Schaufeli \& Leiter, 2001). For example, positive school climate (Lim \& Eo, 2014), organizational support (Ju et al., 2015; Meylan et al., 2015), organizational citizenship (İnandı \& Büyüközkan, 2013) and organizational trust (Van Maele \& Van Houtte, 2015) reduce burnout while student misbehaviors (McCormick \& Barnett, 2011; Aloe et al., 2014) and work stress (Yu et al., 2015) increase burnout. Teacher burnout is an important problem affecting education systems (Loonstra, Brouwers \& Tomic, 2009). Student success relies on effective teachers (Rushton, Morgan \& Richard, 2007; Seidel \& Shavelson, 2007). It seems likely that student success may fall if teachers experience burnout. This study aims to determine levels of burnout experienced by teachers working at public schools, and to determine differences caused by gender, branch, and school type.

The literature on burnout offer some models to explain it. Cherniss (1980) claimed that burnout was a psychological state caused by work stress, social relations, uncertain goals, lack of organizational support, and this state makes workers isolate themselves from the work place. Edelwich and Brodsky (1980) defined burnout as a psychological defense mechanism developed in response to unmet expectations of newbie workers who were enthusiastic, vibrant and idealistic. Perlman and Hartman (1982) portrayed burnout as emotional and physiological exhaustion, low work efficiency and impersonation reactions to stress. According to Meier (1983) burnout occurs

\footnotetext{
${ }^{1}$ Res. Asst., Artvin Çoruh University, Artvin, Turkey, kbozkus@artvin.edu.tr
} 
Bozkuş, K. (2018). The level of burnout experienced by teachers. International Journal of Social Sciences and Education Research, 4(1), 61-67.

once workers expect harm rather than benefit from work when they experience low self-efficacy. Maslach and Jackson (1981) assert that burnout happens in three stages: workers experience emotional exhaustion first when they encounter demands that are hard to fulfill. Then they find desensitization to work as a way to escape from this situation. Finally, they perceive themselves as a failure. Pines (2005) indicates that people who are motivated by work cannot achieve success they expect when they under persistent pressure, and this results in a reduced motivation, depression, despair, physiological, emotional and psychological exhaustion.

\section{Methodology}

The research was conducted employing survey method. This method is based on collecting data from large samples, so generalization of the findings can be meaningful (Fraenkel, Wallen, $\&$ Hyun, 2012). Population of this research consists of 981 teachers working at public schools in Tavşanlı province of Kütahya city in Turkey during 2014-2015 academic year. Data collected through questionnaire forms from 400 teachers working at 25 schools selected randomly from 123 public schools in the province. Forms that are answered faulty were eliminated and remaining 386 forms were analyzed. Minimum sample size required to represent the population was calculated as 350 considering $5 \%$ error rate and $2 \%$ confidence interval. Therefore, the sample of this research can be claimed to be representative. Statistics regarding to participants of the research were indicated in Table 1.

Table 1. Participant statistics

\begin{tabular}{cccc}
\hline \multirow{2}{*}{ Factor } & Variable & Frequency & $\begin{array}{c}\text { Percentage } \\
\text { (\%) }\end{array}$ \\
\hline \multirow{2}{*}{ Gender } & Male & 216 & 56 \\
& Female & 156 & 40 \\
& Unspecified & 14 & 4 \\
\hline \multirow{2}{*}{ Branch } & Class Teacher & 136 & 35 \\
& Subject Teacher & 250 & 65 \\
\hline \multirow{2}{*}{ School Type } & Primary & 142 & 37 \\
& Secondary & 86 & 22 \\
& High & 111 & 29 \\
& Technical High & 47 & 12 \\
\hline
\end{tabular}

The Burnout Measure Short Version developed by Pines (2005) and adapted into Turkish by Tümkaya, Çam and Çavuşoğlu (2009) was used as the data collection instrument. The measure includes 10 items in 7 point Likert style ranging from 'never' and 'always'. Adapted version of the measure explains 55.92\% of total variance in one factor and had Cronbach's alpha reliability coefficient of .91. When data of this study had been analyzed, $63.8 \%$ of total variance were explained in one factor and had Cronbach's alpha reliability coefficient of .90. Also confirmatory factor analysis of data collected in the study indicated an acceptable fit $(\chi 2 / \mathrm{df}=5.66$, RMSEA $=0.11$, GFI $=0.91$, AGFI $=0.85$, SRMR $=0.053$, IFI $=0.97, \mathrm{NFI}=0.96, \mathrm{RFI}=0.95$ ).

\section{Results}

The highest mean belongs to the item 'I feel tired' $(\bar{X}=4.16, \mathrm{Sd}=1.36)$ which located at sometimes level (Table 2). Items of 'I feel disappointed with people' $(\bar{X}=3.41, \mathrm{Sd}=1.41)$, 'I feel hopeless' $(\bar{X}=3.27, \mathrm{Sd}=1.56)$ and 'I've had it' $(\bar{X}=2.78, \mathrm{Sd}=1.38)$ were located at rarely level. Others were located at almost never level. The lowest mean belongs to the item 'I feel worthless and unsuccessful' ( $\bar{X}=2.06, \mathrm{Sd}=1.31)$. 
Bozkuş, K. (2018). The level of burnout experienced by teachers. International Journal of Social Sciences and Education Research, 4(1), 61-67.

Table 2. Item statistics

\begin{tabular}{cccc}
\hline Items & $\overline{\boldsymbol{X}}$ & SD \\
\hline Tired & 4.16 & 1.36 \\
Disappointed with people & 3.41 & 1.41 \\
Hopeless & 3.27 & 1.56 \\
I've had it & 2.78 & 1.38 \\
Depressed & 2.55 & 1.37 \\
Difficulties sleeping & 2.50 & 1.43 \\
Trapped & 2.46 & 1.56 \\
Helpless & 2.38 & 1.43 \\
Physically weak/Sickly & 2.27 & 1.25 \\
Worthless/Like a Failure & 2.06 & 1.31 \\
\hline Total & $\mathbf{2 . 7 9}$ & $\mathbf{1 . 0 2}$ \\
\hline
\end{tabular}

Once levels of teacher burnout were calculated through total means, 214 (55.3\%) teachers were at never and almost never levels, $144(37.2 \%)$ teachers were at rarely and sometimes levels, $28(7.1 \%)$ teachers were at often, very often and always levels (Table 3 ).

Table 3. Burnout levels of teachers

\begin{tabular}{ccc}
\hline Burnout Levels & f & $\mathbf{\%}$ \\
\hline Never & 69 & 17.8 \\
\hline Almost never & 145 & 37.5 \\
\hline Rarely & 99 & 25.6 \\
\hline Sometimes & 45 & 11.6 \\
\hline Often & 23 & 5.9 \\
\hline Very often & 4 & 1 \\
\hline Always & 1 & 0.2 \\
\hline Total & 386 & 100 \\
\hline
\end{tabular}

Table 4. Gender based differences in perceptions

\begin{tabular}{|c|c|c|c|c|c|c|c|}
\hline Item & Gender & $n$ & $\bar{X}$ & $S D$ & $d f$ & $t$ & $p$ \\
\hline \multirow{2}{*}{ Tired } & Male & 216 & 3.95 & 1.38 & \multirow{2}{*}{370} & \multirow{2}{*}{-3.17} & \multirow{2}{*}{$.002 *$} \\
\hline & Female & 156 & 4.40 & 1.30 & & & \\
\hline \multirow{2}{*}{$\begin{array}{l}\text { Disappointed with } \\
\text { people }\end{array}$} & Male & 216 & 3.42 & 1.40 & \multirow{2}{*}{370} & \multirow{2}{*}{0.25} & \multirow{2}{*}{.803} \\
\hline & Female & 156 & 3.38 & 1.39 & & & \\
\hline \multirow{2}{*}{ Hopeless } & Male & 216 & 3.27 & 1.59 & \multirow{2}{*}{370} & \multirow{2}{*}{.07} & \multirow{2}{*}{.94} \\
\hline & Female & 156 & 3.26 & 1.48 & & & \\
\hline \multirow{2}{*}{ Trapped } & Male & 216 & 2.61 & 1.67 & \multirow{2}{*}{370} & \multirow{2}{*}{2.31} & \multirow{2}{*}{$.021 *$} \\
\hline & Female & 156 & 2.24 & 1.38 & & & \\
\hline \multirow{2}{*}{ Helpless } & Male & 216 & 2.43 & 1.46 & \multirow{2}{*}{370} & \multirow{2}{*}{1.05} & \multirow{2}{*}{.295} \\
\hline & Female & 156 & 2.27 & 1.36 & & & \\
\hline \multirow{2}{*}{ Depressed } & Male & 216 & 2.62 & 1.42 & \multirow{2}{*}{370} & \multirow{2}{*}{1.68} & \multirow{2}{*}{.093} \\
\hline & Female & 156 & 2.38 & 1.22 & & & \\
\hline \multirow{2}{*}{$\begin{array}{r}\text { Physically } \\
\text { weak/Sickly }\end{array}$} & Male & 216 & 2.18 & 1.25 & \multirow{2}{*}{370} & \multirow{2}{*}{-1.82} & \multirow{2}{*}{.070} \\
\hline & Female & 156 & 2.42 & 1.26 & & & \\
\hline \multirow{2}{*}{$\begin{array}{l}\text { Worthless/Like a } \\
\text { Failure }\end{array}$} & Male & 216 & 2.19 & 1.37 & \multirow{2}{*}{370} & \multirow{2}{*}{2.36} & \multirow{2}{*}{$.019 *$} \\
\hline & Female & 156 & 1.87 & 1.19 & & & \\
\hline \multirow{2}{*}{$\begin{array}{l}\text { Difficulties sleep- } \\
\text { ing }\end{array}$} & Male & 216 & 2.46 & 1.40 & \multirow{2}{*}{370} & & \\
\hline & Female & 156 & 2.58 & 1.45 & & -0.19 & .430 \\
\hline I've had it & Male & 216 & 2.81 & 1.43 & 370 & 082 & 413 \\
\hline I ve nad it & Female & 156 & 2.69 & 1.27 & $3 / 0$ & 0.82 & .413 \\
\hline Total & Male & 216 & 2.79 & 1.08 & 370 & 0.41 & 681 \\
\hline Total & Female & 156 & 2.75 & .92 & $3 / 0$ & 0.41 & .001 \\
\hline
\end{tabular}

$* \mathrm{p}<.05$ 
Bozkuş, K. (2018). The level of burnout experienced by teachers. International Journal of Social Sciences and Education Research, 4(1), 61-67.

Independent samples $t$ test to define gender based differences indicated differences at the items of 'I feel tired' ( $\mathrm{t}(370)=-3.17, \mathrm{p}<.05)$, 'I feel trapped' $(\mathrm{t}(370)=2.31, \mathrm{p}<.05)$ and 'I feel worthless and unsuccessful' ( $\mathrm{t}(370)=2.36, \mathrm{p}<.05)$. Female teachers had a higher mean at the item of 'I feel tired', and male teachers had higher means at the items of 'I feel trapped' and 'I feel worthless and unsuccessful'. Therefore, it can be asserted that female teachers tired more than male teachers and male teachers felt more trapped, worthless and unsuccessful than female teachers.

Independent samples $t$ test to define teacher branch based differences indicated no differences (Table 5).

Table 5. Teacher Branch Based Differences in Perceptions

\begin{tabular}{|c|c|c|c|c|c|c|c|}
\hline Item & Branch & $n$ & $\bar{X}$ & $S D$ & $d f$ & $t$ & $p$ \\
\hline \multirow{2}{*}{ Tired } & Class & 136 & 4.06 & 1.33 & \multirow{2}{*}{384} & \multirow{2}{*}{-1.09} & \multirow{2}{*}{.278} \\
\hline & Subject & 250 & 4.22 & 1.36 & & & \\
\hline \multirow{2}{*}{$\begin{array}{l}\text { Disappointed with } \\
\text { people }\end{array}$} & Class & 136 & 3.36 & 1.43 & \multirow{2}{*}{384} & \multirow{2}{*}{-0.51} & \multirow{2}{*}{.614} \\
\hline & Subject & 250 & 3.44 & 1.39 & & & \\
\hline \multirow{2}{*}{ Hopeless } & Class & 136 & 3.11 & 1.50 & \multirow{2}{*}{384} & \multirow{2}{*}{-1.53} & \multirow{2}{*}{.127} \\
\hline & Subject & 250 & 3.36 & 1.58 & & & \\
\hline \multirow{2}{*}{ Trapped } & Class & 136 & 2.57 & 1.60 & \multirow{2}{*}{384} & \multirow{2}{*}{1.07} & \multirow{2}{*}{.287} \\
\hline & Subject & 250 & 2.40 & 1.54 & & & \\
\hline \multirow{2}{*}{ Helpless } & Class & 136 & 2.35 & 1.38 & \multirow{2}{*}{384} & \multirow[b]{2}{*}{-0.28} & \multirow[b]{2}{*}{.778} \\
\hline & Subject & 250 & 2.40 & 1.46 & & & \\
\hline \multirow{2}{*}{ Depressed } & Class & 136 & 2.59 & 1.27 & \multirow{2}{*}{384} & \multirow{2}{*}{0.39} & \multirow{2}{*}{.700} \\
\hline & Subject & 250 & 2.53 & 1.41 & & & \\
\hline \multirow{2}{*}{$\begin{array}{r}\text { Physically } \\
\text { weak/Sickly }\end{array}$} & Class & 136 & 2.29 & 1.34 & \multirow{2}{*}{384} & \multirow{2}{*}{0.23} & \multirow{2}{*}{.818} \\
\hline & Subject & 250 & 2.26 & 1.20 & & & \\
\hline \multirow{2}{*}{$\begin{array}{l}\text { Worthless/Like a } \\
\text { Failure } \\
\end{array}$} & Class & 136 & 2.07 & 1.35 & \multirow{2}{*}{384} & \multirow{2}{*}{0.02} & \multirow{2}{*}{.988} \\
\hline & Subject & 250 & 2.06 & 1.28 & & & \\
\hline \multirow{2}{*}{$\begin{array}{l}\text { Difficulties sleep- } \\
\text { ing }\end{array}$} & Class & 136 & 2.50 & 1.47 & \multirow{2}{*}{384} & & \\
\hline & Subject & 250 & 2.50 & 1.41 & & -0.03 & .979 \\
\hline I'yo had it & Class & 136 & 2.66 & 1.35 & & & \\
\hline I ve had it & Subject & 250 & 2.85 & 1.39 & 384 & -1.27 & .207 \\
\hline Total & Class & 136 & 2.76 & 1.04 & 384 & & 678 \\
\hline I otal & Subject & 250 & 2.80 & 1.01 & 384 & -0.42 & $.0 / 8$ \\
\hline
\end{tabular}

ANOVA test to determine school type differences indicated statistically significant differences at the items of 'I feel disappointed with people' $(\mathrm{F}(3,382)=4.79, \mathrm{p}<.05)$, 'I feel hopelessness' $(\mathrm{F}(3,382)=6.19, \mathrm{p}<.05)$, 'I feel trapped' $(\mathrm{F}(3,382)=5.34, \mathrm{p}<.05)$, 'I feel helpless' $(\mathrm{F}(3,382)=$ $5.55, \mathrm{p}<.05)$ and at total scores $(\mathrm{F}(3,382)=4.05, \mathrm{p}<.05)$. LSD post hoc test to determine resource of differences indicated that mean scores of teachers working at technical high schools were higher at the items and total scores given above. Therefore, it can be asserted that teachers working at technical high schools feel more disappointment, hopelessness, as trapped, as helpless and they experience burnout more than teachers working at primary, middle and other high schools. 
Bozkuş, K. (2018). The level of burnout experienced by teachers. International Journal of Social Sciences and Education Research, 4(1), 61-67.

Table 6. School type based differences in perceptions

\begin{tabular}{|c|c|c|c|c|c|c|c|}
\hline Item & School & $n$ & $\overline{\boldsymbol{X}}$ & $S D$ & $F$ & $\bar{p}$ & $L S D$ \\
\hline \multirow{4}{*}{ Tired } & Primary & 142 & 4.08 & 1.32 & \multirow{4}{*}{1.58} & \multirow{4}{*}{.194} & \\
\hline & Secondary & 86 & 4.29 & 1.55 & & & \\
\hline & High & 111 & 4.03 & 1.21 & & & \\
\hline & Technical & 47 & 4.47 & 1.36 & & & \\
\hline \multirow{4}{*}{$\begin{array}{l}\text { Disappointed } \\
\text { people }\end{array}$} & Primary & 142 & 3.37 & 1.43 & \multirow{4}{*}{4.79} & \multirow{4}{*}{$.003 *$} & $1-4$ \\
\hline & Secondary & 86 & 3.34 & 1.34 & & & $2-4$ \\
\hline & High & 111 & 3.22 & 1.28 & & & $3-4$ \\
\hline & Technical & 47 & 4.11 & 1.55 & & & \\
\hline \multirow{4}{*}{ Hopeless } & Primary & 142 & 3.14 & 1.49 & \multirow{4}{*}{6.19} & \multirow{4}{*}{$.000 *$} & $1-4$ \\
\hline & Secondary & 86 & 3.10 & 1.53 & & & $2-4$ \\
\hline & High & 111 & 3.20 & 1.54 & & & $3-4$ \\
\hline & Technical & 47 & 4.17 & 1.56 & & & \\
\hline \multirow{4}{*}{ Trapped } & Primary & 142 & 2.55 & 1.59 & \multirow{4}{*}{5.34} & \multirow{4}{*}{$.001 *$} & $1-4$ \\
\hline & Secondary & 86 & 2.19 & 1.47 & & & $2-4$ \\
\hline & High & 111 & 2.24 & 1.41 & & & $3-4$ \\
\hline & Technical & 47 & 3.19 & 1.75 & & & \\
\hline \multirow{4}{*}{ Helpless } & Primary & 142 & 2.35 & 1.36 & \multirow{4}{*}{5.55} & \multirow{4}{*}{$.001 *$} & $1-4$ \\
\hline & Secondary & 86 & 2.12 & 1.32 & & & $2-4$ \\
\hline & High & 111 & 2.31 & 1.34 & & & $3-4$ \\
\hline & Technical & 47 & 3.13 & 1.77 & & & \\
\hline \multirow{4}{*}{ Depressed } & Primary & 142 & 2.59 & 1.26 & \multirow{4}{*}{2.19} & \multirow{4}{*}{.089} & \\
\hline & Secondary & 86 & 2.28 & 1.33 & & & \\
\hline & High & 111 & 2.57 & 1.41 & & & \\
\hline & Technical & 47 & 2.89 & 1.52 & & & \\
\hline \multirow{4}{*}{$\begin{array}{l}\text { Physically } \\
\text { weak/Sickly }\end{array}$} & Primary & 142 & 2.26 & 1.33 & \multirow{4}{*}{0.24} & \multirow{4}{*}{.868} & \\
\hline & Secondary & 86 & 2.31 & 1.22 & & & \\
\hline & High & 111 & 2.20 & 1.21 & & & \\
\hline & Technical & 47 & 2.36 & 1.18 & & & \\
\hline \multirow{4}{*}{$\begin{array}{l}\text { Worthless/Like } \\
\text { Failure }\end{array}$} & Primary & 142 & 2.05 & 1.33 & \multirow{4}{*}{2.49} & & \\
\hline & Secondary & 86 & 1.87 & 1.11 & & 060 & \\
\hline & High & 111 & 2.05 & 1.25 & & .060 & \\
\hline & Technical & 47 & 2.51 & 1.57 & & & \\
\hline & Primary & 142 & 2.51 & 1.45 & & & \\
\hline Difficulties sleening & Secondary & 86 & 2.57 & 1.36 & 015 & 925 & \\
\hline & High & 111 & 2.43 & 1.44 & 0.15 & .925 & \\
\hline & Technical & 47 & 2.53 & 1.50 & & & \\
\hline & Primary & 142 & 2.68 & 1.33 & & & \\
\hline I've had it & Secondary & 86 & 2.64 & 1.29 & 2.14 & 094 & \\
\hline I ve nad il & High & 111 & 2.84 & 1.46 & 2.14 & .094 & \\
\hline & Technical & 47 & 3.21 & 1.42 & & & \\
\hline & Primary & 142 & 2.76 & 1.02 & & & $1-4$ \\
\hline Total & Secondary & 86 & 2.67 & 0.93 & 405 & $007 *$ & $2-4$ \\
\hline & High & 111 & 2.71 & 1.01 & & & $3-4$ \\
\hline & Technical & 47 & 3.25 & 1.07 & & & \\
\hline
\end{tabular}

${ }^{*} \mathrm{p}<.05,1:$ Primary, 2:Secondary, 3:High, 4:Technical

\section{Discussion and recommendations}

A great number of teachers of the research sample felt tired. Burnout based on tiredness is often associated to work load (Van Droogenbroeck, Spruyt \& Vanroelen, 2014). Female teachers tired more than their male colleagues and male teachers feel trapped, worthless and unsuccessful 
Bozkuş, K. (2018). The level of burnout experienced by teachers. International Journal of Social Sciences and Education Research, 4(1), 61-67.

more than their female colleagues. This difference may be resulted from females' being better at social relations comparing to males (Ergin, 1992). Also, literature indicate similar findings regarding to gender based differences in burnout (Başol \& Altay, 2009; Çağlar, 2011; Deryakulu, 2005; Otacioğlu, 2008; Özben \& Argun, 2003). Likewise, 44.7\% of the teachers experienced burnout at rarely and higher levels. Given the fact that burnout reduces teacher efficiency and student success this finding can be considered as alerting. Similar rates had been reported by past research (Akın \& Oğuz, 2010; Akman, Taşkın, Özden \& Çörtü, 2010; Bağcı \& Karagül, 2013; Çağlar, 2011). There was no statistically significant difference affecting class teachers' and subject teachers' perceptions regarding burnout. This might stem from the fact that burnout is related to work place factors and both teacher groups experience similar work environments. Teachers working at technical high schools experience burnout more than those work at other schools. Given the fact that face to face interrelationships at technical high schools take place at a higher rate comparing to other types of schools, the difference might be a result of this situation. Because burnout is experienced more frequently in where social relations are intense (Maslach \& Jackson, 1981; Maslach, 2003).

Recommendations generated from the findings of this study are as follows: Workloads of teachers should be reduced due to the fact that increased work load may result in increased burnout. Given the fact that almost half of the teachers experience burnout, it is essential to research why teachers experience burnout, and then generate preventive measures to cure the problem. International studies may provide clues in preventing teacher burnout. Similarly investigating why teachers working at technical high schools experience burnout more than their counterparts working at other high schools is vital to finding solutions.

\section{Reference}

Akın, U. \& Oğuz, E. (2010). Öğretmenlerin işkoliklik ve tükenmişlik düzeylerinin ilişkisi ve çeşitli değişkenler açısından incelenmesi. Kuram ve Uygulamada Eğitim Yönetimi, 16(3), 309-327.

Akman, B., Taşkın, N., Özden, Z., \& Çörtü, F. (2010). Okul öncesi öğretmenlerinde tükenmişlik üzerine bir çalışma. Illköğretim Online, 9(2), 807-815.

Aloe, A. M., Shisler, S. M., Norris, B. D., Nickerson, A. B., \& Rinker, T. W. (2014). A multivariate metaanalysis of student misbehavior and teacher burnout. Educational Research Review, 12, 30-44.

Bağcı, H. \& Karagül, S. (2013). Türkçe öğretmenlerinin mesleki tükenmişlik düzeyi. Mehmet Akif Ersoy Üniversitesi Sosyal Bilimler Enstitüsü Dergisi, 5(8), 184-193.

Başol, G. \& Altay, M. (2009). Eğitim yöneticisi ve öğretmenlerin mesleki tükenmişlik düzeylerinin incelenmesi. Kuram ve Uygulamada Ĕ̆itim Yönetimi, 15(58), 191-216.

Cherniss, C. (1980). Staff burnout: Job stress in the human services. Thousand Oaks: Sage.

Çağlar, Ç. (2011). Okullardaki örgütsel güven düzeyi ile öğretmenlerin mesleki tükenmişlik düzeyinin bazı değişkenler açısından incelenmesi. Kuram ve Uygulamada Eğitim Bilimleri, 11(4), 1827-1847.

De Heus, P. \& Diekstra, R. F. W. (1999). Do teachers burn out more easily? A comparison of teachers with other social professions on work stress and burnout symptoms. İçinde R. Vandenberghe \& A. Michael Huberman (Eds.), Understanding and preventing teacher burnout: A sourcebook of international research and practice. Cambridge: Cambridge University Press.

Deryakulu, D. (2005). Bilgisayar öğretmenlerinin tükenmişlik düzeylerinin incelenmesi. Eurasian Journal of Educational Research, 19, 35-53.

Edelwich, J. \& Brodsky, A. (1980). Burn-Out: Stages of disillusionment in the helping professions. New York: Human Sciences Press.

Ergin, C. (1992). Doktor ve hemşirelerde tükenmişlik ve Maslach tükenmişlik ölçeği’nin uyarlanması. 7. Ulusal Psikoloji Kongresi Bilimsel Çalışmaları. Ankara: Hacettepe Üniversitesi. 
Bozkuş, K. (2018). The level of burnout experienced by teachers. International Journal of Social Sciences and Education Research, 4(1), 61-67.

Fraenkel, J. R., Wallen, N., \& Hyun, H. (2012). How to design and evaluate research in education (8. b.). New York: McGraw-Hill.

Freudenberger, H. J. (1974). Staff burn-out. Journal of Social Issues, 30(1), 159-165.

İnand,, Y. \& Büyüközkan, A. S. (2013). The effect of organizational citizenship behaviours of primary school teachers on their burnout. Educational Sciences: Theory \& Practice, 13(3), 1545-1550.

Ju, C., Lan, J., Li, Y., Feng, W., \& You, X. (2015). The mediating role of workplace social support on the relationship between trait emotional intelligence and teacher burnout. Teaching and Teacher Education, $51,58-67$.

Lim, S. \& Eo, S. (2014). The mediating roles of collective teacher efficacy in the relations of teachers' perceptions of school organizational climate to their burnout. Teaching and Teacher Education, 44, 138-147.

Loonstra, B., Brouwers, A., \& Tomic, W. (2009). Feelings of existential fulfillment and burnout among secondary school teachers. Teaching and Teacher Education, 25(5), 752-757.

Maslach, C. (2003). Burnout: The cost of caring. Los Altos: Malor Books.

Maslach, C. \& Jackson, S. E. (1981). The measurement of experienced burnout. Journal of Occupational Behavior, 2, 99-113.

Maslach, C. \& Jackson, S. E. (1986). Maslach burnout inventory. Palo Alto: Consulting Psychologists.

Maslach, C., Schaufeli, W. B., \& Leiter, M. P. (2001). Job burnout. Annual Review of Psychology, 52, 397422.

McCormick, J. \& Barnett, K. (2011). Teachers' attributions for stress and their relationships with burnout. International Journal of Educational Management, 25(3), 278-293.

Meier, S. T. (1983). Toward a theory of burnout. Human Relations, 36(10), 899-910.

Meylan, N., Doudin, P. A., Curchod-Ruedi, D., \& Stephan, P. (2015). School burnout and social support: The importance of parent and teacher support. Psychologie Française, 60, 1-15.

Otacığlu, S. G. (2008). Müzik öğretmenlerinde tükenmişlik sendromu ve etkileyen faktörler. İnönü Üniversitesi Ĕ̆itim Fakültesi Dergisi, 9(15), 103-116.

Özben, Ş. \& Argun, Y. (2003). İlköğretim öğretmenlerinin umutsuzluk ve tükenmişlik düzeyleri üzerine bir araştırma. Ege Eğitim Dergisi,3(1), 36-48.

Perlman, B. \& Hartman, E. A. (1982). Burnout: Summary and future research. Human Relations, 35(4), 283-305.

Pines, A. M. (2005). The burnout measure, short version. International Journal of Stress Management, $12(1), 78-88$.

Rushton, S., Morgan, J., \& Richard, M. (2007). Teacher's Myers-Briggs personality profiles: Identifying effective teacher personality traits. Teaching and Teacher Education, 23, 432-441.

Seidel, T. \& Shavelson, R. (2007). Teaching effectiveness research in the past decade: The role of theory and research design in disentangling meta-analysis results. Review of Educational Research, 77, 454499.

Schaufeli, W. B. \& Enzmann, D. (1998). The burnout companion to study and practice: A critical analysis. London: Taylor \& Francis.

Tümkaya, S., Çam, S., \& Çavuşoğlu, İ. (2009). Tükenmişlik ölçeği kısa versiyonu'nun Türkçe’ye uyarlama, geçerlik ve güvenirlik çalışması. Çukurova Üniversitesi Sosyal Bilimler Enstitüsü Dergisi, 18(1), 387 398.

Van Droogenbroeck, F., Spruyt, B., \& Vanroelen, C. (2014). Burnout among senior teachers: Investigating the role of workload and interpersonal relationships at work. Teaching and Teacher Education, 43, 99109.

Van Maele, D. \& Van Houtte, M. (2015). Trust in school: a pathway to inhibit teacher burnout? Journal of Educational Administration, 53(1), 93-115.

Yu, X., Wang, P., Zhai, X., Dai, H., \& Yang, Q. (2015). The effect of work stress on job burnout among teachers: The mediating role of self-efficacy. Social Indicators Research, 122, 701-708. 